\title{
Redundancy Level Impact of the Mean Time to Failure on Wireless Sensor Network
}

\author{
Alaa E. S. Ahmed \\ ${ }^{1}$ College of Computer and Information Sciences, \\ Al Imam Mohammad Ibn Saud Islamic University (IMSIU) \\ Riyadh, Kingdom of Saudi Arabia \\ ${ }^{2}$ Department of Electrical Engineering, \\ Faculty of Engineering (Shoubra), Benha University, Egypt
}

\author{
Mostafa E. A. Ibrahim \\ ${ }^{1}$ College of Computer and Information Sciences, \\ Al Imam Mohammad Ibn Saud Islamic University (IMSIU) \\ Riyadh, Kingdom of Saudi Arabia \\ ${ }^{2}$ Communication and Computer Engineering Dept, \\ Benha Faculty of Engineering, Benha University, Egypt
}

\begin{abstract}
Recently, wireless sensor networks (WSNs) have gained a great attention due to their ability to monitor various environments, such as temperature, pressure sound, etc. They are constructed from a large number of sensor nodes with computation and communication abilities. Most probably, sensors are deployed in an uncontrolled environment and hence their failures are inevitable all times of work. Faulty sensor nodes may cause incorrect sensing data, wrong data computation or even incorrect communication. Achieving a reliable wireless sensor networks is a most needed goal to ensure quality of service whether at deployment time or during normal operation. While Nodes redundancy is considered as an effective solution to overcome nodes failures, it may negatively affect the WSN lifetime. Redundancy may lead to more energy drains of the whole system. In this paper, the impact of redundancy level on the Mean Time to Failure (MTTF) of a clustered based wireless Sensor Networks (WSNs) is investigated. An expression that can be used to determine the most suitable redundancy level that maximizes the network MTTF is derived and evaluated.
\end{abstract}

Keywords-Wireless sensor network; reliability; clustering; fault tolerant; Mean Time to Failure (MTTF)

\section{INTRODUCTION}

Wireless sensor networks (WSNs) are currently being considered for many communications applications, such as environmental monitoring, agricultural monitoring, machine health monitoring, surveillance, and medical monitoring. These networks provide a great ability to better understand the surrounding physical phenomenon and translate their effect to be analyzed to give a more accurate way to control our surroundings. However, there are many research QoS challenges that need to be studied and analyzed to achieve scalable reliable and dependable networks.

Traditionally, redundancy is a technique belongs to replica management approaches which are designed to overcome nodes failure and to achieve reliable system. Redundancy most probably leads to maximize the availability of the nodes.

In sensor networks, sensor nodes can fail due to many reasons. Among those reasons are: battery power depletion, hardware problem and cruel environmental circumstances in which the node is running. Nods are failed according to a variable failure rate that start by a low value and continuously increase and may reach to value that cause a complete failure to the system. One solution to overcome nodes failure is to use reliable hardware that safely send, receive and store data. This solution is expensive solution that contradicts with the objective of having cheap wireless sensor. Replicating data on spatially separated nodes is a good method for minimized the failure probability. Traditional solutions for replica systems are to use a pre-determined fixed number of replicas [1].

Because of the dynamic behavior of failure, selecting a suitable redundancy level is an important issue. In some cases small number of replicas is better and on the other hand in some situations higher number of replicas is more suitable. Because of that, the process of selecting the suitable redundancy level should be adaptive to achieve less energy consumption and higher degree of availability. One way to determine the most suitable redundancy level is to have a complete knowledge of the network which is not a practical solution and doesn't cope with scalability issue. Also, it requires much information to be circulated around the network to keep up to date with the current situation which consumes more energy. The solution is to find a solution to determine the most suitable redundancy level that achieves a reliable request while maximizing the Mean Time to Failure MTTF of the system. The contribution in this paper is as follows:

1) Describe a system model that captures all stages that affect the energy consumption 1 of entire the network.

2) Propose an expression that is used to determine the most suitable redundancy level to maximize the mean time to failure MTTF.

3) Provide a numerical data analysis that implements the proposed model to show the tradeoff between the energy consumption and the reliability improvement.

The remainder of the paper is organized as follows: In Section 2, the related work is introduced. The system model assumptions are presented in Section 3. Section 4 shows the analysis and numerical results. Finally, the paper ends with the conclusion in Section 5.

\section{RELATED WORK}

The general problem of achieving reliable WSN has been covered intensively in literature. Providing the concept of redundancy is considered an efficient trend in order to achieve reliable wireless sensor network. Redundancy types can be 
subdivided into three categories, spatial redundancy, physical redundancy and temporal redundancy.

Spatial redundancy is based on geographically distribute the sensor nodes over the field area in such a way that guarantee the replication of the resources all over the coverage area. Spatial redundancy is suitable for WSN since the information read by any sensor node is available and stored in other different nodes. This kind of redundancy can be used in implementing fault tolerant approached to improve the WSN reliability level. Spatial redundancy is drawn by large number of metrics [2], [3]. In [4] authors employed the graph theory and cut sets in order to define the redundancy degree of the wireless sensor network. It is the most possible number of nodes that its disappearance doesn't affect the accuracy of the measured data.

Physical redundancy is a hardware redundancy and is considered one of the easiest ways to achieve reliable systems. It is mainly based on having a dense number of independent sensor nodes to be distributed and cover certain filed area [5], [6].

Temporal redundancy is mostly used to get better accuracy of the sensor nodes readings and to overcome the transient failures. In Wireless sensor networks, the reading come from any single node may be not precise. The solution is to improve the whole system reliability by using multiple reading from the same sensor node [7].

In [8] authors present a method for evaluating the system reliability by generating a fault tree for the whole network at the time of failure occurrence. The provided solution is independent of the underlying network technology. However, the model doesn't take the energy consumption into consideration.

In [9], [10] a power consumption saving approach is introduced. Power consumption saving is considered one way of achieving reliable wireless sensor network. The approach adopts the use of Colored Petri Net to model the network and provide a good way to be integrated with different applications.

In [11] authors present an efficient method that could be used in transmitting the data through the WSN. The method is mainly tends to minimize the number of lost packets by using multiples paths for data transmissions to overcome either the node or the path failure. One problem with this method is the bottleneck produced as a result of having more than one path per packets.

Clustering is one of the most used techniques in reducing the energy consumption in WSNs and consequently increase the sensor node life time. One of the earliest WS communication algorithms is LEACH [12]. It stands for Low Energy Adaptive Clustering Hierarchy Algorithm. The existence of clustering leads to minimize the energy usage with the cluster but at the same time accelerate the energy consumption of the cluster head $\mathrm{CH}$. To solve this problem, the concept of balanced clustering is introduced in [13]. Another cluster based approach called Distributed Weight-Based Energy-Efficient Hierarchical Clustering (DWEHC) is introduced in [14]. It is based on forming well balanced clusters by adopting the hierarchical level of a node depending with the ability to minimize the energy requirements by taking into consideration two factors, the range of a cluster and the path to the $\mathrm{CH}$.

In [15] energy an Energy-Efficient Unequal Clustering (EEUC) mechanism is introduced. It is based on dividing the nodes into unequal cluster sized. This technique is dynamic in the sense that, it picks any node randomly and checks whether it lies within the competitive radius or not. So, the node would join the most suitable cluster that saves its energy. A lot of energy-efficient clustering algorithms can be found is available in [16].

\section{SYSTEM MODEL ASSUMPTIONS}

In this section, we describe the proposed system model and its assumptions. The network is formed by a collection of limited-energy Sensor Nodes (SNs) scattered randomly on a certain geographical area. The WSN model used in this work is adopted from [17].

1) Initially each sensor node has an initial energy $E_{\text {init }}$.

2) The sensor nodes are scattered randomly through a square field area.

3) It is assumed that each node has failure probability $f(t)$ where (where $0<\mathrm{f}(\mathrm{t})<1$ ).

4) $\mathrm{LEACH}$ algorithm [11] is used for routing and managing the network clustering. First the cluster head " $\mathrm{CH}$ " is elected per cluster. The $\mathrm{CH}$ is responsible for administrating the communication within the cluster, aggregate data read by sensors nodes, and set up the data sent to the sink nodes. This algorithm runs in the form of rounds. Due to the extra responsibilities of the $\mathrm{CH}$ node, it is exposed to lose their energy quicker than other nodes. For this reason, the $\mathrm{CH}$ role is rotated around all member of the cluster according to a certain probability $\mathrm{p}$. the value of $\mathrm{p}$ depends on the cluster size.

5) In this model it is assumed that the communication channels are reliable and never fail.

6) It is assumed that the reliability of the system is expressed as follow [18].

$$
\begin{gathered}
\mathrm{R}_{m-o f-n}=\sum_{i=0}^{N-M}\left(\begin{array}{c}
N \\
\mathrm{i}
\end{array}\right)(1-R(t))^{i} R(t)^{N-i} \\
=\sum_{i=M}^{N}\left(\begin{array}{c}
N \\
\mathrm{i}
\end{array}\right) R(t)^{i}(1-R(t))^{N-i} \\
\text { Where, } \quad\left(\begin{array}{c}
N \\
\mathrm{i}
\end{array}\right)=\frac{\mathrm{N} !}{\mathrm{i} !(\mathrm{N}-\mathrm{i}) !}
\end{gathered}
$$
cluster

Where, $\mathrm{N}$ is the number of identical sensor nodes per

$\mathrm{m}=$ the number of functional nodes

$\mathrm{R}(\mathrm{t})=$ the reliability of individual module.

$\mathrm{R}_{\mathrm{m}-\mathrm{of}-\mathrm{N}(\mathrm{t})}$ is the reliability of that $(\mathrm{N}-\mathrm{m})$ or fewer nodes have failed by time $\mathrm{t}$ (or - at least $\mathrm{m}$ are functional).

7) In response to a request, a sensor node sends a message to its $\mathrm{CH}$ node. It is assumed that the number of hops between 
the sensor node and the cluster head is $\mathrm{N}_{\text {clust }}$. Any request needs " $m$ " sensor nodes to reach a certain redundancy level. The total energy $E_{t}$ for any request has two components $E_{1}$ and $E_{2}$. The first is the energy consumed to send the data from sensor node to the $\mathrm{CH}$ and the other is the energy consumed by the WSN to transmit sensor data from $\mathrm{CH}$ to the sink node. The total energy equation can be expressed as follow [17]:

$$
\mathrm{E}_{\mathrm{t}}=\mathrm{E}_{1}+\mathrm{E}_{2}
$$

$$
\mathrm{E}_{\mathrm{t}}=\left[\mathrm{N}_{\text {hop }}\left(\mathrm{E}_{\text {receive }}+\mathrm{E}_{\text {transmit }}\right)\right]+\left[\mathrm{m}\left(\mathrm{E}_{\text {receive }}+\mathrm{E}_{\text {transmit }}\right)+\mathrm{N}_{\text {clust }}\right.
$$$$
\left.\left(\mathrm{E}_{\mathrm{i}}\right)\right]
$$

8) The energy consumed in executing the clustering process includes the amount of energy due to multicasting the leadership announcement message and the energy due to joining the cluster. If $\mathrm{p}$ is the probability of becoming a $\mathrm{CH}$, there will be $\mathrm{p}^{*} \mathrm{~N}_{\text {clust }}$ sensor node that multicast the leadership announcement message which in turn is forwarded by each sensor to the next hop until covering the whole nodes in the cluster. Assume the number of hops per cluster is Nhop/cluster Thus, the energy required for broadcasting is [17]:

$$
\mathrm{E}_{\text {br }}=\mathrm{pN}_{\text {clust }}\left(\mathrm{E}_{\text {receive }}+\mathrm{E}_{\text {transmit }}\right)+\mathrm{N}_{\text {hop/cluster }}\left(\left(\mathrm{E}_{\text {receive }}+\mathrm{E}_{\text {transmit }}\right)\right.
$$

9) The energy consumed due to the cluster-join process includes the energy consumed by any sensor node to inform the $\mathrm{CH}$ about their cluster membership and the energy consumed by the $\mathrm{CH}$ to send back an acknowledge message to the sensor nodes.

$$
\mathrm{E}_{\text {join }}=\mathrm{N}_{\text {clust }}\left(\mathrm{E}_{\text {receive }}+\mathrm{E}_{\text {transmit }}\right) \text {. }
$$

10) Since the clustering process is executed a number of rounds " $\mathrm{N}_{\text {round }}$ " then the total energy consumed in clustering is expressed as [17]:

$$
E_{\text {clustering }}=N_{\text {round }}\left[E_{b r}+E_{\text {join }}\right]
$$

\section{SYSTEM MTTF}

The main objective here is to achieve the most suitable redundancy level $\mathrm{m}$ that achieves a reliable request while maximizing the Mean Time to Failure MTTF. Assume it is required to process a request with required reliability $\mathrm{Rq}$. Assume $\lambda$ is the failure rate of different sensor nodes. Since we use $\mathrm{m}$ out of $\mathrm{N}$ reliability model, the MTTF can be calculated according to the following:

Since, $\quad$ MTTF $_{\mathrm{m} \text { of } \mathrm{N}}=\int_{0}^{\infty} \mathrm{R}_{\mathrm{m} \text { of } \mathrm{n}}$

By substituting from (1):

$$
\begin{aligned}
& \text { MTTF }_{\mathrm{m} \text { of } \mathrm{N}}=\int_{0}^{\infty}\left[\sum_{\mathrm{i}=\mathrm{m}}^{\mathrm{N}}\left(\begin{array}{l}
\mathrm{N} \\
\mathrm{i}
\end{array}\right) \mathrm{R}(\mathrm{t})^{\mathrm{i}}(1-\mathrm{R}(\mathrm{t}))^{\mathrm{N}-\mathrm{i}}\right] \mathrm{dt} \\
& \mathrm{MTTF}_{\mathrm{m} \text { of } \mathrm{N}}=\int_{0}^{\infty}\left[\sum_{\mathrm{i}=\mathrm{m}}^{\mathrm{N}}\left(\begin{array}{l}
\mathrm{N} \\
\mathrm{i}
\end{array}\right) \mathrm{e}^{-\mathrm{i} \lambda \mathrm{t}}\left(1-\mathrm{e}^{-\lambda \mathrm{t}}\right)^{\mathrm{N}-\mathrm{i}}\right] \mathrm{dt}
\end{aligned}
$$

By introducing $v=e^{-\lambda t}$ we obtain $d v=\lambda e^{-\lambda t} d t \rightarrow$ $d t=\frac{d v}{\lambda e^{-\lambda t}} d v$
By substituting in the MTTF $m$ of $n$

$$
\begin{gathered}
\mathrm{MTTF}_{\mathrm{m} \text { of } \mathrm{N}}=\sum_{\mathrm{i}=\mathrm{m}}^{\mathrm{N}}\left(\begin{array}{l}
\mathrm{N} \\
\mathrm{i}
\end{array}\right) \int_{0}^{1} \frac{\mathrm{v}^{\mathrm{i}}(1-\mathrm{v})^{\mathrm{N}-\mathrm{i}} \mathrm{dv}}{\lambda \mathrm{v}} \\
\mathrm{MTTF}_{\mathrm{m} \text { of } \mathrm{N}}=\sum_{\mathrm{i}=\mathrm{m}}^{\mathrm{N}}\left(\begin{array}{l}
\mathrm{N} \\
\mathrm{i}
\end{array}\right) \frac{1}{\lambda} \int_{0}^{1}\left[\mathrm{v}^{\mathrm{i}-1}(1-\mathrm{v})^{\mathrm{N}-\mathrm{i}}\right] \mathrm{dv}
\end{gathered}
$$

According to Gamma and Beta function definition [18]:

So,

$$
\int_{0}^{1}\left[v^{i-1}(1-v)^{N-i}\right] d v=\frac{\Gamma(i) \Gamma(n-i+1)}{\Gamma(n+1)}
$$

$$
\mathrm{MTTF}_{\mathrm{m} \text { of } \mathrm{N}}=\sum_{\mathrm{i}=\mathrm{m}}^{\mathrm{N}}\left(\begin{array}{l}
\mathrm{N} \\
\mathrm{i}
\end{array}\right) \frac{1}{\lambda} \frac{\Gamma(\mathrm{i}) \Gamma(\mathrm{n}-\mathrm{i}+1)}{\Gamma(\mathrm{n}+1)}
$$

And consequently,

$$
\mathrm{MTTF}_{\mathrm{m} \text { of } N}=\sum_{\mathrm{i}=\mathrm{m}}^{\mathrm{N}}\left(\begin{array}{l}
\mathrm{N} \\
\mathrm{i}
\end{array}\right) \frac{1}{\lambda} \frac{(\mathrm{i}-1) !(\mathrm{n}-\mathrm{i}) !}{(\mathrm{n}) !}
$$

Then,

$$
\mathrm{MTTF}_{\mathrm{m} \text { of } N}=\frac{1}{\lambda} \sum_{\mathrm{i}=\mathrm{m}}^{\mathrm{N}} \frac{\mathrm{n} !}{\mathrm{i} !(\mathrm{n}-\mathrm{i}) !} \frac{(\mathrm{i}-1) !(\mathrm{n}-\mathrm{i}) !}{(\mathrm{n}) !}
$$

Finally,

$$
\mathrm{MTTF}_{\mathrm{m} \text { of } \mathrm{N}}=\frac{1}{\lambda} \sum_{i=m}^{N} \frac{1}{\mathrm{i}}
$$

\section{ANALYSIS AND NUMERICAL RESUlTS}

In this section we introduce the numeric data analysis that implements the proposed model to show the tradeoff between the energy consumption and the reliability improvement. Also, we show the effect of changing $m$ on the MTTF of the system. The following paragraph indicates parameters used along with their default parameters. These default values are similar to the values used in [33]. The WSN model consists of 400 sensor nodes distributed randomly in a square area of $200 \mathrm{~m}$ by $200 \mathrm{~m}$. Each sensor node has an initial energy of 10 Joule. The energy consumed per bit due to the transmitter/receiver radio process$\mathrm{E}_{\text {receive }} \& \mathrm{E}_{\text {transmit }}$ - is $50 \mathrm{~nJ} / \mathrm{bit}$.

\section{A. Reliability and MTTF Analysis}

Fig. 1 shows the relation between the reliability and the redundancy level $\mathrm{m}$ under the environment parameters described in the previous paragraph. It is possible to use other parameter but still the overall trend is the same. In previous chapter the reliability analysis is based on adopting and applying the $\mathrm{N}$ out of $\mathrm{m}$ redundant systems to achieve a reliable WSN. The $\mathrm{N}$ sensor nodes are assumed to be identical 
in all configurations. The $\mathrm{N}$ out of $\mathrm{m}$ redundant system is considered a sort of parallel system where $m$ represents the minimum number of sensors per cluster required to have a fully functional sensing operation during certain time period $\mathrm{T}$. we assume that sensor failure may occur according to a failure rate $\lambda=.001$ and $\mathrm{T}=200$ unit time. In case $\mathrm{m}=1$ the system is reduced to a fully parallel system since any single node will do the job. On the other hand when $\mathrm{m}=\mathrm{N}$, this means that the system is act as a series system since the all members in the cluster are required to have a correct operation. From that Fig. 1 shows the relation between the system reliability and the $\mathrm{m}$ value through out a certain period of time according to (1). The graph show that as the $\mathrm{m}$ value increases the reliability level is reduced. This result consistent with the fact that says as $\mathrm{m}$ increase the system tends to converge to a series one. The overall reliability of series systems is lower than the reliability of each individual component. At the same time as the $m$ value decrease the system tends to converge to be a parallel one that ha reliability an improved reliability level.

As a consequence of the previous result, Fig. 2 shows the relation between the $m$ value and the MTTF (8). The result shows that the MTTF is decreased as $m$ increase. This result is compatible with the result achieved in Fig. 1. As the system tends to be fully parallel, the MTTF increase since the reliability increases.

The following two figures (Fig. 3 and 4) shows the relation between both the system reliability and the MTTF and the m value but with failure rate $=.0001$ and $\mathrm{T}=200$ unit time. The figure shows the same trend achieved in Fig. 1 and 2.

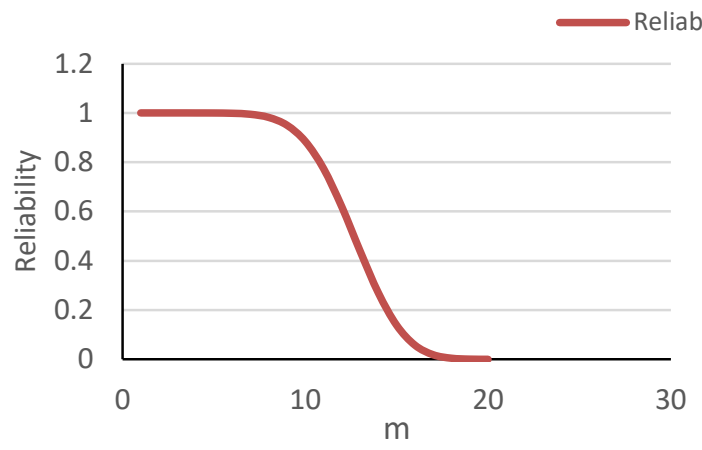

Fig. 1. Reliability VSm with $\lambda=.001$.

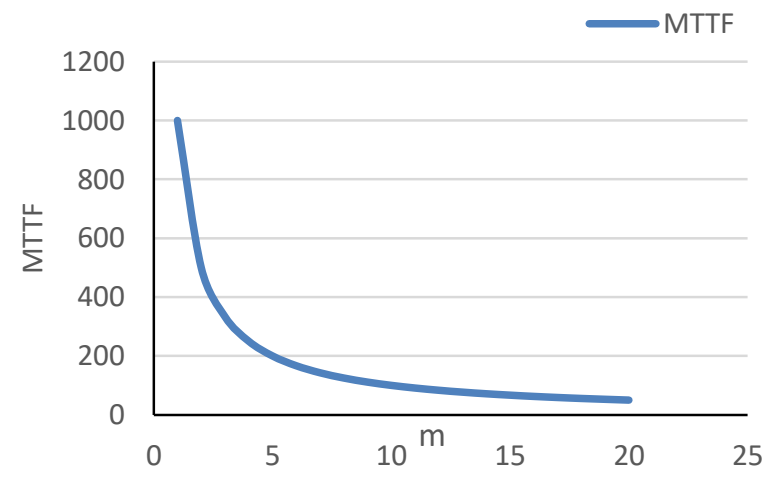

Fig. 2. MTTF VS $m$ with $\lambda=.001$.

\section{B. Energy Level Consumed Analysis}

In this section we introduce the numerical analysis related to the level of consumed energy of due to applying the m out of $\mathrm{n}$ redundant system. Fig. 5 shows the relation between the energy consumed as a result of 24 bits size request. The figure shows three curves that represent the consumed energy level with different value of the number of intermediate nodes within the cluster $\left(\mathrm{N}_{\text {clust }}\right)$. As the value of $\mathrm{m}$ increase the energy consumed increase. This result is compatible with the fact that as $\mathrm{m}$ increase, the system tends to work as a series fashion that involve more sensor nodes in the process of completing the request. As the number of involved sensors increase the energy consumed will increase.

Also, Fig. 6 shows the relation between the $m$ value and the consumed energy in the case of changing the parameter of the number of nodes exists between the cluster head and the sink node $\left(\mathrm{N}_{\text {hop }}\right)$. The result indicates that as $\mathrm{m}$ value increase the energy consumed increase even with different values of $\mathrm{N}_{\text {hop. }}$.

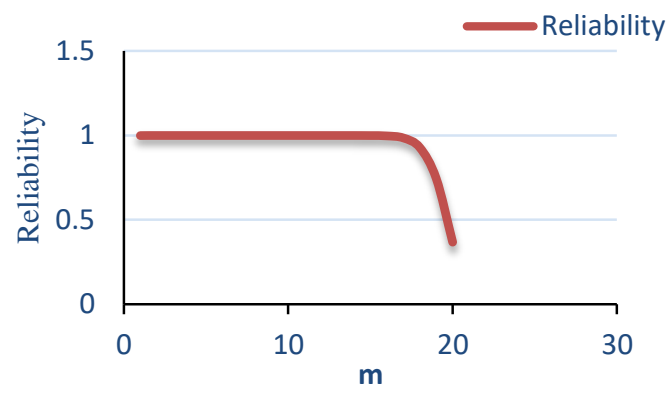

Fig. 3. Reliability VS $m$ with $\lambda=.0001$.

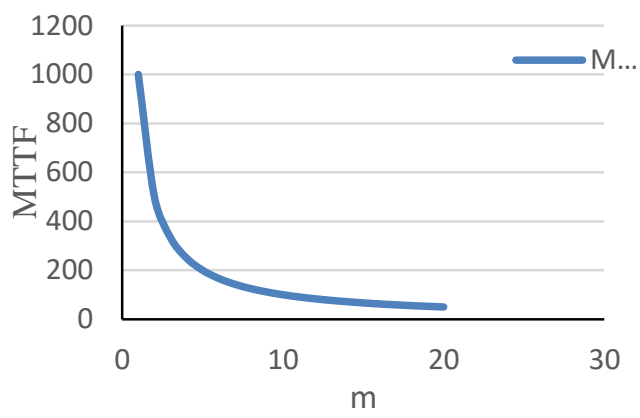

Fig. 4. MTTF VS $m$ with $\lambda=.0001$.

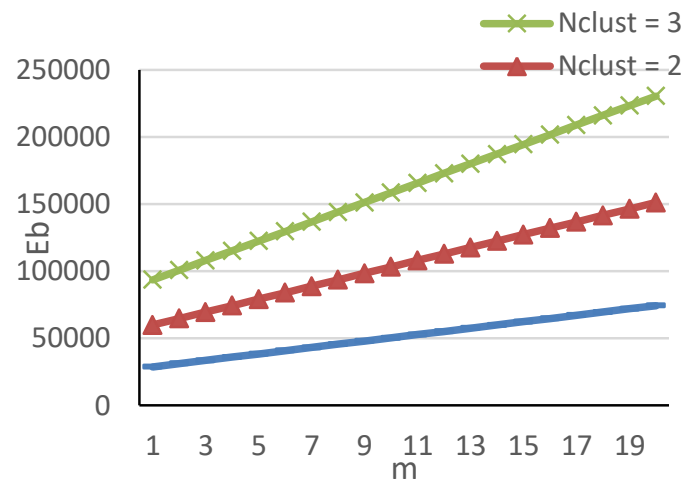

Fig. 5. 5Eb VS m with $\lambda=.001$. 


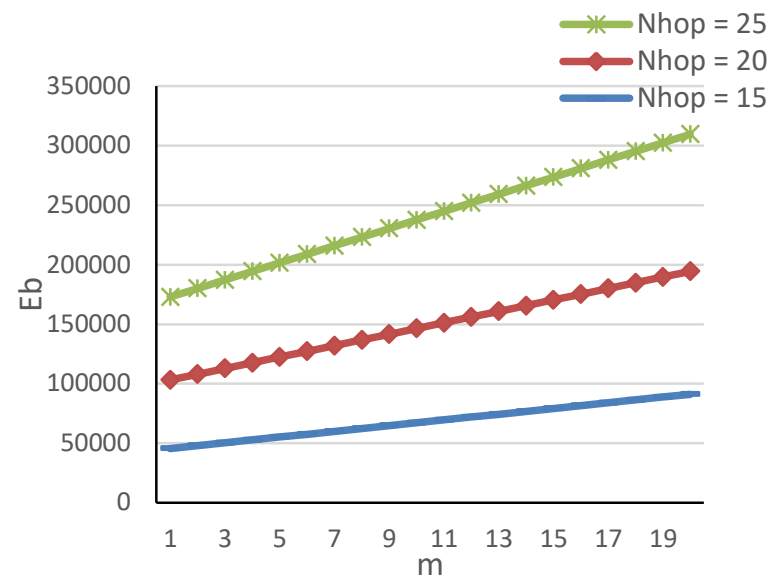

Fig. 6. $\mathrm{E}_{\mathrm{t}}$ VS $\mathrm{m}$ with $\lambda=.0001$.

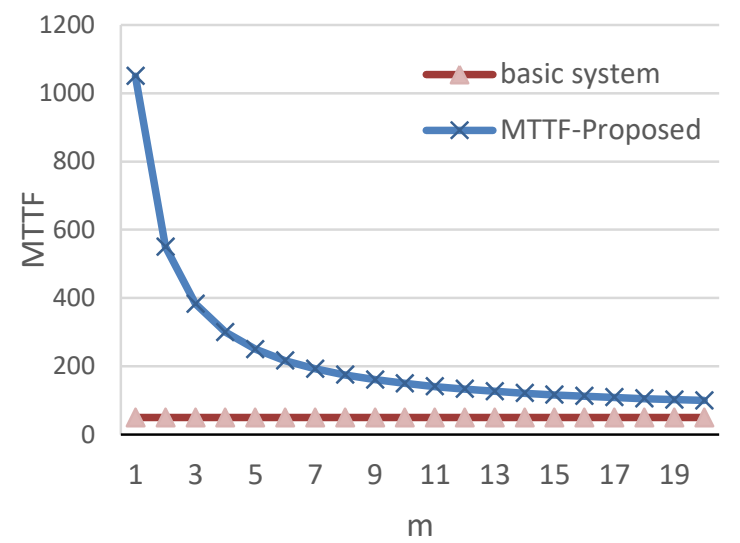

Fig. 7. MTTF VS $m$ with $\lambda=.001$.

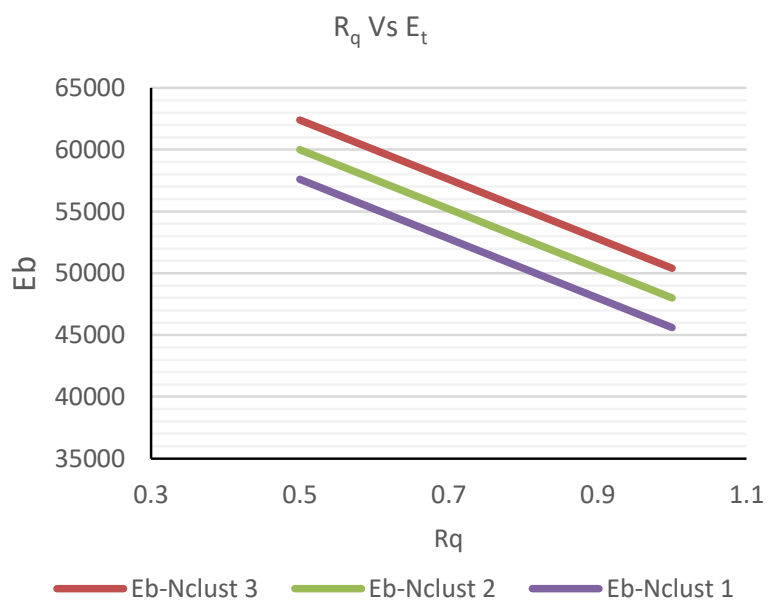

Fig. 8. Et vs $\mathrm{Rq}$ with $\lambda=.001$.

C. Comparison with the Basic System $(m=1)$

In this section we compare the MTTF for both proposed design and the basic system in which there is no redundancy at all. Fig. 7 shows the change of the MTTF in both the basic system and the proposed design. We notice that the proposed system give higher system MTTF compared with the basic design under the set of assumed parameter values characterizing the WSN.

\section{Consumed Energy Effect on the Required Reliability Level}

One of the important parameters that is useful to the WSN designer is to be able to determine the energy consumed Et when a required level of reliability $R_{\mathrm{q}}$ is needed to be satisfied. Fig. 8 shows the relation between the $\mathrm{R}_{\mathrm{q}}$ and Et under the set of assumed parameter values characterizing the WSN.

According to the proposed model, as the required the reliability level increase the number of active nodes per cluster $\mathrm{m}$ decrease and that would cause the energy consumed to decrease. This result is shown in Fig. 8. That would consequently help the system designer is able to have a tentative estimation about the energy consumed for the required and needed system reliability level. One more result shown in the figure is that as the number of intermediate nodes within the cluster $\mathrm{N}_{\text {clust }}$ increase the energy consumed per certain required reliability increase.

\section{CONCLUSIONS}

In this paper, a probability model to calculate the system reliability, the energy Consumption of a request, and, consequently, the system MTTF is introduced. Throughout the model we considered energy consumed by the sensor nodes due to request processing, periodic status update processing and cluster formation. The proposed model is adopting a group communication service represented of forming, managing and using the cluster. The effect of the redundancy level on MTTF is evaluated. A series of numerical analysis result that show and validate the proposed model showing the reliability, MTTF and energy consumed in order to achieve a dependent WSN system are conducted. As a conclusion, the MTTF of the system increase as the number of active components $m$ per cluster decrease. Also, the $\mathrm{m}$ parameter could be used as design parameter that helps the designer to achieve a system with a required level of reliability.

\section{REFERENCES}

[1] M. A. Mohamed, W. K. Seah and I. Welch, "Reliability in wireless sensor networks: A survey and challenges ahead" Computer Networks, vol 79, pp. 166-187, Elsevier, 2015.

[2] G. Jaber, R. Kacimi and Z. Mammeri "Exploiting redundancy for energy-efficiency in Wireless Sensor Networks" in ninth Wireless and Mobile Networking Conference, IEEE, 2016.

[3] C. Volosencu, D. Pescaru, L. Jurca and A. Doboli "Redundancy and Its Applications in Wireless Sensor Networks: A Survey", WSEAS Transactions on Computers, Vol. 8, no. 4, pp. 705-714, April 2009.

[4] B. Rachid , H. Hafid , k. Bouabdellah "A distributed approach using redundancy for wireless sensor networks reconfiguration" Models \& Optimisation and Mathematical Analysis Journal vol. 01, pp. 84-90, 2012.

[5] D. Shim, C. Yang, "Method of detecting and isolating fault in redundant sensors, and method of accommodating fault in redundant sensors using the same", USPTO Application, Nov. 2008.

[6] Z. Bojkovic and B. Bakmaz "A survey on wireless sensor networks deployment", WSEAS Transactions on Communications, vol 7, no 12, pp. 1172-1181, Dec. 2008. 
[7] G. Jesus, A. Casimiro and A. Oliveira "A Survey on Data Quality for Dependable Monitoring in Wireless Sensor Networks" Sensors ,vol. 17, no. 9, pp. 1-23, 2017.

[8] I. Silva, L.A Guedes, P. Portugal, F. Vasques "Reliability and availability evaluation of wireless sensor networks for industrial applications" Sensors, vol. 12, pp. 806-838, 2012.

[9] A.V.L Dâmaso, D. Freitas, N.S Rosa, B, Silva, P.R.M Maciel "Evaluating the power consumption of wireless sensor network applications using models" Sensors, vol. 3, pp. 3473-3500, 2013.

[10] A.V.L Dâmaso, N.S Rosa, P.R.M Maciel, "Using coloured petri net for evaluating the power consumption of wireless sensor network" Int. J. Distrib. Sens. Netw. (IJDSN) 2014.

[11] W. Fang, F. Liu, F. Yang, L. Shu, and S. Nishio, "Energy-efficient cooperative communication for data transmission in wireless sensor networks," IEEE Transactions on Consumer Electronics, vol. 56, no. 4, november 2010.

[12] S. Xia, M. Tan, Z. Zhao and T Xiang "Improvement for LEACH Algorithm in Wireless Sensor Network" International Conference of Young Computer Scientists, Engineers and Educators, pp 287-298, Springer, 2016.
[13] S. A. Nikolidakis, D. Kandris, D.D Vergados, C. Douligeris "Energy efficient routing in wireless sensor networks through balanced clustering" Algorithms, vol. 6, no. 1, pp. 29-42, 2013.

[14] P. Ding, J. Holliday, A. Celik, "Distributed computing in sensor systems" Distributed energy-efficient hierarchical clustering for wireless sensor networks; vol. 3560, pp. 322-339. Berlin, Germany: Springer; 2005.

[15] G. Chen, C. Li, M. Ye, J. Wu "An unequal cluster-based routing protocol in wireless sensor networks" Wireless Networks, vol. 15 no. 2, pp 193-207, 2009.

[16] B. M. Vallapuram, G. P. Nair, K. Thangamani, "A convivial energy based clustering (CEBC) solution for lifetime enhancement of wireless sensor networks", Artificial intelligence and evolutionary algorithms in engineering systems, pp. 225-234, Springer; 2015.

[17] A. E. S. Ahmed, "Analytical modeling for reliability in cluster based wireless sensor networks" in Ninth International Conference on Computer Engineering and Systems (ICCES). IEEE, 20-25, 2014.

[18] M. Rausand “System Reliability Theory”. 2nd edition, Wiley, 2003. 